\title{
Proving Termination of Input-Consuming Logic Programs
}

\author{
Jan-Georg Smaus* \\ INRIA-Rocquencourt \\ BP 105, 78153 Le Chesnay Cedex, France
}

\begin{abstract}
A class of predicates is identified for which termination does not depend on left-to-right execution. The only assumption about the selection rule is that derivations are input-consuming, that is, in each derivation step, the input arguments of the selected atom do not become instantiated. This assumption is a natural abstraction of previous work on programs with delay declarations. The method for showing that a predicate is in that class is based on level mappings, closely following the traditional approach for LDderivations. Programs are assumed to be well and nicely moded, which are two widely used concepts for verification. Many predicates terminate under such weak assumptions. Knowing these predicates is useful even for programs where not all predicates have this property.
\end{abstract}

\section{Introduction}

Termination of logic programs has been widely studied for LD-derivations, that is derivations where the leftmost atom in a query is always selected $[1,3$, $7,8,9,10,12]$. These works are based on the following idea: when an atom $a$ in a query is selected, it is possible to pin down the size $e^{1}$ of $a$. This size cannot change via further instantiation. It is then shown that for the atoms introduced in this derivation step, it is again possible to pin down their size when eventually they are selected, and these atoms are smaller than $a$.

This idea has also been applied to arbitrary derivations [6]. Since no restriction is imposed on when an atom can be selected, it is required that in each query in a derivation, the size of each atom is always bounded. Programs that fulfill this requirement are called strongly terminating. The class of strongly terminating programs is very limited.

For most logic programs, it is necessary for termination to require a certain degree of instantiation of an atom before it can be selected. This can be achieved using delay declarations $[2,16,17,18,19,22,23]$. The problem is that, depending on what kind of delay declarations and selection rule are used, it is often not possible to pin down the size of the selected atom, since this size may depend on the resolution of other atoms in the query that are

\footnotetext{
${ }^{*}$ Formerly: University of Kent at Canterbury, United Kingdom.

${ }^{1}$ The technical meaning of "pinning down the size" differs among different methods. This will be discussed in Sect. 7 .
} 
not yet resolved. Nevertheless, the approaches by Marchiori and Teusink [17] and Martin and King [18], and to a limited extent Lüttringhaus-Kappel [16] are based on the idea described above.

Our approach falls between the two extremes of making no assumptions about the selection rule on the one hand and making very specific assumptions on the other. We believe that a reasonable minimal requirement for termination can be formulated in terms of modes:

In each derivation step, the input arguments of the selected atom cannot become instantiated.

In other words, an atom in a query can only be selected when it is sufficiently instantiated so that the most general unifier (MGU) with the clause head does not bind the input arguments of the atom. We call derivations which meet this requirement input-consuming.

This paper is about identifying predicates for which all input-consuming derivations are finite. Other works in this area have usually made specific assumptions about the selection rule and the delay declarations, for example local selection rules [17], delay declarations that test arguments for groundness or rigidness $[16,18]$, or the default left-to-right selection rule of most Prolog implementations $[19,22,23]$. In contrast, we show how previous results about LD-derivations can be generalised, the only assumption about the selection rule being that derivations are input-consuming.

We exploit that under certain conditions, it is enough to rely on a relative decrease in the size of the selected atom.

Example 1.1 Consider the usual append program, where the first two argument positions are input positions. The following is an input-consuming derivation. The selected atom is always underlined. On the right hand side, we indicate some of the variable bindings made in this derivation.

$$
\begin{aligned}
& \text { append([1], [], As), append(As, [], Bs }) \leadsto \\
& \text { append([], [],As' }) \text {, append }\left(\left[1 \mid \mathrm{As} \mathbf{s}^{\prime}\right],[], \mathrm{Bs}\right) \leadsto
\end{aligned}
$$

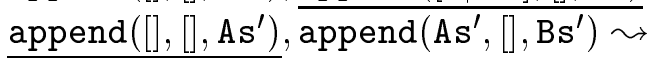

$$
\begin{aligned}
& \overline{\text { append }\left([],[], \mathrm{Bs}^{\prime}\right)} \leadsto \square \\
& \begin{array}{r}
\left(\mathrm{As}=\left[1 \mid \mathrm{As}^{\prime}\right]\right) \\
\left(\mathrm{Bs}=\left[1 \mid \mathrm{Bs}^{\prime}\right]\right) \\
\left(\mathrm{As}^{\prime}=[]\right) \\
\left(\mathrm{Bs}^{\prime}=[]\right)
\end{array}
\end{aligned}
$$

When append([1|A $\left.\left.\mathbf{s}^{\prime}\right],[], \mathrm{Bs}\right)$ is selected, it is not possible to pin down its size in any meaningful way. In fact, nothing can be said about the length of the (input-consuming) derivation associated with append([1|A $\left.\left.\mathbf{s}^{\prime}\right],[], \mathrm{Bs}\right)$ without knowing about other atoms which might instantiate $\mathbf{A s}^{\prime}$. However, the derivation could be infinite only if some derivation associated with append([], [], As' was infinite. Our method is based on such a dependency between the atoms of a query.

As discussed in Sect. 7 , previous approaches $[6,16,17,18]$ cannot formally show termination of derivations with coroutining such as the one above.

Even though the class of programs for which all input-consuming derivations are finite is obviously larger than the class of strongly terminating programs, it is still quite limited. The following example illustrates this. 
of a clause in $P$ and $\sigma$ an MGU of $p(\mathbf{s}, \mathbf{t}) \theta$ and $p(\mathbf{v}, \mathbf{u})$. We call $p(\mathbf{s}, \mathbf{t}) \theta$ the selected atom and $R \theta \sigma$ the resolvent of $Q \theta$ and $h \leftarrow B$. A derivation step is input-consuming if $\operatorname{dom}(\sigma) \cap \operatorname{var} s(\mathbf{s} \theta)=\emptyset .^{2}$

A derivation $\xi$ for a program $P$ is a sequence $\left\langle Q_{0}, \theta_{0}\right\rangle ;\left\langle Q_{1}, \theta_{1}\right\rangle ; \ldots$ where each pair $\left\langle Q_{i}, \theta_{i}\right\rangle ;\left\langle Q_{i+1}, \theta_{i+1}\right\rangle$ in $\xi$ is a derivation step. Alternatively, we also say that $\xi$ is a derivation of $P \cup\left\{Q_{0} \theta_{0}\right\}$. We sometimes denote a derivation as $Q_{0} \theta_{0} ; Q_{1} \theta_{1} ; \ldots$ An $\mathbf{L D}$-derivation is a derivation where the selected atom is always the leftmost atom in a query. An input-consuming derivation is a derivation consisting of input-consuming derivation steps.

If $(F, a, H) ;(F, B, H) \theta$ is a step in a derivation, then each atom in $B \theta$ is a direct descendant of $a$, and $b \theta$ is a direct descendant of $b$ for all $b \in F, H$. We say $b$ is a descendant of $a$ if $(b, a)$ is in the reflexive, transitive closure of the relation is a direct descendant. The descendants of a set of atoms are defined in the obvious way. Consider a derivation $Q_{0} ; \ldots ; Q_{i} ; \ldots ; Q_{j} ; Q_{j+1} ; \ldots$ We call $Q_{j} ; Q_{j+1}$ an $a$-step if $a$ is an atom in $Q_{i}$ and the selected atom in $Q_{j} ; Q_{j+1}$ is a descendant of $a$.

\section{Modes}

In this section we introduce well moded and nicely moded programs, which are standard concepts used for verification of logic programs $[2,5,11,12,13]$.

Well-modedness has been introduced by Dembinski and Małuszyński [11] and widely used since. In Mercury it is even mandatory that programs are well moded (possibly after reordering of atoms by the compiler), which is one of the reasons for its remarkable performance [24].

Definition 3.1 [well moded] A query $Q=p_{1}\left(\mathbf{s}_{1}, \mathbf{t}_{1}\right), \ldots, p_{n}\left(\mathbf{s}_{n}, \mathbf{t}_{n}\right)$ is well moded if for all $i \in\{1, \ldots, n\}$ and $L=1$

$$
\operatorname{vars}\left(\mathbf{s}_{i}\right) \subseteq \bigcup_{j=L}^{i-1} \operatorname{vars}\left(\mathbf{t}_{j}\right)
$$

The clause $p\left(\mathbf{t}_{0}, \mathbf{s}_{n+1}\right) \leftarrow Q$ is well moded if (1) holds for all $i \in\{1, \ldots, n+$ $1\}$ and $L=0$. A program is well moded if all of its clauses are well moded.

Note that a one-atom query $p(\mathbf{s}, \mathbf{t})$ is well moded if and only if $\mathbf{s}$ is ground.

Another widely used concept is the following.

Definition 3.2 [nicely moded] A query $Q=p_{1}\left(\mathbf{s}_{1}, \mathbf{t}_{1}\right), \ldots, p_{n}\left(\mathbf{s}_{n}, \mathbf{t}_{n}\right)$ is nicely moded if $\mathbf{t}_{1}, \ldots, \mathbf{t}_{n}$ is a linear vector of terms and for all $i \in\{1, \ldots, n\}$

$$
\operatorname{vars}\left(\mathbf{s}_{i}\right) \cap \bigcup_{j=i}^{n} \operatorname{vars}\left(\mathbf{t}_{j}\right)=\emptyset .
$$

\footnotetext{
${ }^{2}$ Since the MGU is unique up to variable renaming, we may assume that whenever possible, an MGU $\sigma$ is used such that $\operatorname{dom}(\sigma) \cap \operatorname{var} s(\mathbf{s} \theta)=\emptyset$.
} 
The clause $C=p\left(\mathbf{t}_{0}, \mathbf{s}_{n+1}\right) \leftarrow Q$ is nicely moded if $Q$ is nicely moded and

$$
\operatorname{vars}\left(\mathbf{t}_{0}\right) \cap \bigcup_{j=1}^{n} \operatorname{vars}\left(\mathbf{t}_{j}\right)=\emptyset .
$$

A program is nicely moded if all of its clauses are nicely moded.

Note that a one-atom query $p(\mathbf{s}, \mathbf{t})$ is nicely moded if and only if $\operatorname{var} s(\mathbf{s}) \cap$ $\operatorname{vars}(\mathbf{t})=\emptyset$ and $\mathbf{t}$ is linear. We can thus state the following proposition which follows from the definitions.

Proposition 3.1 A one-atom query $p(\mathbf{s}, \mathbf{t})$ is well and nicely moded if and only if $\mathbf{s}$ is ground and $\mathbf{t}$ is linear.

Example 3.1 The program in Ex. 1.2 is well and nicely moded in mode \{permute $(I, O)$, delete $(I, O, O)\}$. It is neither well moded nor nicely moded in mode $\{$ permute $(O, I)$, delete $(O, I, I)\}$, however it can easily be made well and nicely moded by interchanging the two body atoms in the second clause.

The example shows that multiple modes of a predicate can be obtained by maintaining multiple (renamed) versions of a predicate, which differ in the order of atoms in the clause bodies. This is why some authors assume that each predicate has a fixed mode $[12,19,24]$. However, in those works, assuming a fixed mode is, from a formal point of view, a real restriction.

In this paper, assuming a fixed mode for each predicate is not at all a restriction. It is merely for notational convenience that we assume, in all formal statements, a "left-to-right" data flow in the above definitions. Our results generalise to multiple modes without having multiple versions of each predicate, since we consider derivations where the textual position of an atom within a query is irrelevant for its selection. For reasons of space, we cannot explain this in more detail, and refer to [20, Subsect. 5.3].

The following lemmas state persistence properties of well-modedness and nicely-modedness.

Lemma 3.2 Every resolvent of a well moded query $Q$ and a well moded clause $C$, where $\operatorname{vars}(C) \cap \operatorname{vars}(Q)=\emptyset$, is well moded [2, Lemma 16].

Lemma 3.3 Every resolvent of a nicely moded query $Q$ and a nicely moded clause $C$, where $\operatorname{vars}(C) \cap \operatorname{var} s(Q)=\emptyset$ and the head of $C$ is input-linear, is nicely moded [2, Lemma 11].

For input-consuming derivations, the requirement that the clause head is input-linear can be dropped. It is assumed that the selected atom is sufficiently instantiated, so that a multiple occurrence of the same variable in the input arguments of the clause head cannot cause any bindings to the query. Note that requiring input-linear clause heads is a severe restriction since it rules out input arguments of the selected atom being tested for equality. 
Lemma 3.4 Every resolvent of a nicely moded query $Q$ and a nicely moded clause $C$, where the derivation step is input-consuming and $\operatorname{var} s(C) \cap$ $\operatorname{vars}(Q)=\emptyset$, is nicely moded. (Proof see [21].)

For a nicely moded program and query, it is guaranteed that every inputconsuming derivation step only instantiates other atoms in the query that occur to the right of the selected atom.

Lemma 3.5 Let $P$ be a nicely moded program, $Q=Q_{1}, p(\mathbf{s}, \mathbf{t}), Q_{2}$ a nicely moded query, and $\langle Q, \emptyset\rangle ;\left\langle Q_{1}, B, Q_{2}, \sigma\right\rangle$ an input-consuming derivation step. Then $\operatorname{dom}(\sigma) \cap \operatorname{vars}\left(Q_{1}\right)=\emptyset$.

Proof. Since the derivation step is input-consuming, $\operatorname{dom}(\sigma) \cap \operatorname{vars}(Q) \subseteq$ $\operatorname{vars}(\mathbf{t})$. Thus since $Q$ is nicely moded, $\operatorname{dom}(\sigma) \cap \operatorname{var} s\left(Q_{1}\right)=\emptyset$.

This section mainly served the purpose of recalling some well-known mode concepts. However, Lemma 3.4 is an original result.

\section{Controlled Coroutining}

In this section we define atom-terminating predicates. A predicate $p$ is atomterminating if (under certain conditions) all input-consuming derivations of a query $p(\mathbf{s}, \mathbf{t})$ are finite. Like Etalle et al. [12], we then show that termination for one-atom queries implies termination for arbitrary queries.

For LD-derivations, it is almost obvious that it is sufficient to show termination for one-atom queries, and it only requires that programs and queries are well moded [12, Lemma 4.2]. Given an LD-derivation $\xi$ for a query $a_{1}, \ldots, a_{n}$, the sub-derivations for each $a_{i}$ do not interleave, and therefore $\xi$ can be regarded as a derivation for $a_{1}$ followed by a derivation for $a_{2}$ and so forth. The following example illustrates that in the context of interleaving sub-derivations (coroutining), this is by no means obvious.

Example 4.1 Consider the usual append program

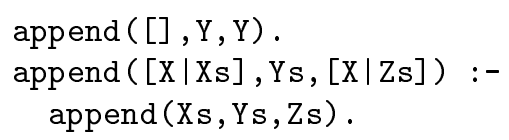

in mode append $(I, I, O)$ and the query

$$
\text { append([], [], As), append([1|As], [], Bs), append(Bs, [], As ). }
$$

This query is well moded but not nicely moded. Then we have the following infinite input-consuming derivation:

$$
\begin{aligned}
& \text { append([], [], As), append }([1 \mid \mathrm{As}],[], \mathrm{Bs}), \operatorname{append}(\mathrm{Bs},[], \mathrm{As}) \leadsto \\
& \text { append([], [], As), } \operatorname{append}\left(\mathrm{As},[], \mathrm{Bs}^{\prime}\right), \operatorname{append}\left(\left[1 \mid \mathrm{Bs}^{\prime}\right],[], \mathrm{As}\right) \leadsto \\
& \operatorname{append}\left([],[],\left[1 \mid \mathrm{As}^{\prime}\right]\right), \operatorname{append}\left(\left[1 \mid \mathrm{As}^{\prime}\right],[], \mathrm{Bs}^{\prime}\right), \operatorname{append}\left(\mathrm{Bs}^{\prime},[], \mathrm{As}^{\prime}\right) \leadsto \ldots
\end{aligned}
$$

This well-known termination problem of programs with coroutining has been identified as circular modes [19]. 
To avoid the problem, we require programs and queries to be nicely moded. Recall that by Prop. 3.1, a one-atom query $p(\mathbf{s}, \mathbf{t})$ is well and nicely moded if and only if $\mathbf{s}$ is ground and $\mathbf{t}$ is linear.

Definition 4.1 [atom-terminating predicate/atom] Let $P$ be a well and nicely moded program. A predicate $p$ in $P$ is atom-terminating if for each well and nicely moded query $p(\mathbf{s}, \mathbf{t})$, all input-consuming derivations of $P \cup$ $\{p(\mathbf{s}, \mathbf{t})\}$ are finite. An atom is atom-terminating if its predicate is atomterminating.

The following lemma says that an atom-terminating atom cannot proceed indefinitely unless it is repeatedly fed by some other atom. It is similar to [22, Lemma 4.2]. For space reasons, we cannot state the precise differences, but note that here, we do not require that clause heads are input-linear. There is a lemma [20, Lemma 6.2] which subsumes [22, Lemma 4.2] and Lemma 4.1, but using this lemma would complicate this paper considerably.

Lemma 4.1 Let $P$ be a well and nicely moded program and $F, b, H$ a well and nicely moded query where $b$ is an atom-terminating atom. An inputconsuming derivation of $P \cup\{F, b, H\}$ can have infinitely many $b$-steps only if it has infinitely many $a$-steps, for some $a \in F$. (Proof see [21].)

The following theorem is a consequence of Lemma 4.1 and states that atomterminating atoms on their own cannot produce an infinite derivation.

Theorem 4.2 Let $P$ be a well and nicely moded program and $Q$ a well and nicely moded query. An input-consuming derivation of $P \cup\{Q\}$ can be infinite only if it contains infinitely many steps where an atom is resolved that is not atom-terminating. (Proof see [21].)

Theorem 4.2 provides us with the formal justification for restricting our attention to one-atom queries. Thus the question is how it can be shown that a predicate is atom-terminating.

\section{Showing that a Predicate is Atom-Terminating}

Termination proofs usually rely, more or less explicitly, on measuring the size of the input in a query $[1,3,7,8,9,10,12]$. We agree with Etalle et al. [12] that it is reasonable to make this dependency explicit. This gives rise to the concept of moded level mapping [12], which is an instance of level mapping [6]. $\mathbf{B}_{P}$ denotes the set of ground atoms using predicates occurring in $P$.

Definition 5.1 [moded level mapping] Let $P$ be a program. |.| is a moded level mapping if

1. it is a level mapping, that is a function $||:. \mathbf{B}_{P} \leadsto \mathbb{N}$, 
2. for any $\mathbf{t}$ and $\mathbf{u},|p(\mathbf{s}, \mathbf{t})|=|p(\mathbf{s}, \mathbf{u})|$.

For $a \in \mathbf{B}_{P},|a|$ is the level of $a$.

Thus the level of an atom only depends on the terms in the input positions.

The following concept is useful for proving termination for a whole program incrementally, by proving it for one predicate at a time [1].

Definition 5.2 [depends on] Let $p, q$ be predicates in a program $P$. We say $p$ refers to $q$ if there is a clause in $P$ with $p$ in its head and $q$ in its body, and $p$ depends on $q$ (written $p \sqsupseteq q)$ if $(p, q)$ is in the reflexive, transitive closure of refers to. We write $p \sqsupset q$ if $p \sqsupseteq q$ and $q \nsupseteq p$, and $p \approx q$ if $p \sqsupseteq q$ and $q \sqsupseteq p$.

Abusing notation, we shall also use the above symbols for atoms, where $p(\mathbf{s}, \mathbf{t}) \sqsupseteq q(\mathbf{u}, \mathbf{v})$ stands for $p \sqsupseteq q$, and likewise for $\sqsupset$ and $\approx$. Furthermore, we denote the equivalence class of a predicate $p$ with respect to $\approx$ as $[p]_{\approx}$.

The following definition provides us with a criterion to prove that a predicate is atom-terminating.

Definition 5.3 [ICD-acceptable] Let $P$ be a program and |.| a moded level mapping. A clause $C=h \leftarrow B$ is acceptable for input-consuming derivations (with respect to |.|) if for every substitution $\theta$ such that $C \theta$ is ground, and for every $a \in B$ such that $a \approx h$, we have $|h \theta|>|a \theta|$. We abbreviate acceptable for input-consuming derivations by ICD-acceptable.

A set of clauses is ICD-acceptable with respect to |.| if each clause is ICD-acceptable with respect to |.|.

Let us compare this concept with some similar concepts in the literature: recurrent [6], well-acceptable [12] and acceptable [4, 10] programs.

Like Decorte and De Schreye [10] and Etalle et al. [12] but unlike Apt and Pedreschi [4] and Bezem [6], we require $|h \theta|>|a \theta|$ only for atoms $a$ where $a \approx h$. This is consistent with the idea that termination should be proven incrementally: to show termination for a predicate $p$, it is assumed that all predicates $q$ with $p \sqsupset q$ have already been shown to terminate. Therefore we can restrict our attention to the predicates $q$ where $q \approx p$.

Like Bezem but unlike Apt and Pedreschi, Decorte and De Schreye and Etalle et al., our definition does not involve models or computed answer substitutions. Traditionally, the definition of acceptable programs is based on a model $M$ of the program, and for a clause $h \leftarrow a_{1}, \ldots, a_{n}, \quad|h \theta|>$ $\left|a_{i} \theta\right|$ is only required if $M \models\left(a_{1}, \ldots, a_{i-1}\right) \theta$. The reason is that for LDderivations, $a_{1}, \ldots, a_{i-1}$ must be completely resolved before $a_{i}$ is selected. By the correctness of LD-resolution [15] and well-modedness [5], the accumulated answer substitution $\theta$, just before $a_{i}$ is selected, is such that $\left(a_{1}, \ldots, a_{i-1}\right) \theta$ is ground and $M=\left(a_{1}, \ldots, a_{i-1}\right) \theta$.

Such considerations count for little when derivations are merely required to be input-consuming. This is illustrated in Ex. 1.2. In the third line of 
the derivation, permute $\left(\left[1 \mid \mathrm{Z}^{\prime \prime}\right], \mathrm{X}^{\prime}\right)$ is selected, although there is no instance of delete $\left([], \mathrm{U}^{\prime}, \mathrm{Z}^{\prime \prime}\right)$ in the model of the program. This problem has been described by saying that delete makes a speculative output binding [19, 23].

Theorem 5.1 Let $P$ be a well and nicely moded program and $p$ be a predicate in $P$. Suppose all predicates $q$ with $p \sqsupset q$ are atom-terminating, and all clauses defining predicates $q \in[p]_{\approx}$ are ICD-acceptable. Then $p$, and hence every predicate in $[p]_{\approx}$, is atom-terminating. (Proof see [21].)

Obviously the above theorem applies in particular if there exists no $q$ such that $p \sqsupset q$, in which case trivially all predicates $q$ with $p \sqsupset q$ are atomterminating.

Example 5.1 We now give a few examples. We denote the term size of a term $t$, that is the number of function and constant symbols that occur in $t$, as $\operatorname{TSize}(t)$.

The clauses defining append $(I, I, O)(\mathrm{Ex} .4$.1) are ICD-acceptable, where $\mid$ append $\left(s_{1}, s_{2}, t\right) \mid=T \operatorname{Size}\left(s_{1}\right)$. Thus append $(I, I, O)$ is atom-terminating. The same holds for append $(O, O, I)$, defining $\mid$ append $\left(t_{1}, t_{2}, s\right) \mid=T \operatorname{Size}(s)$.

The clauses defining delete $(I, O, O)$ (Ex. 1.2) are ICD-acceptable, where $\mid$ delete $\left(s, t_{1}, t_{2}\right) \mid=T \operatorname{Size}(s)$. Thus delete $(I, O, O)$ is atom-terminating. The same holds for delete $(O, I, I)$, defining $\left|\operatorname{delete}\left(t, s_{1}, s_{2}\right)\right|=\operatorname{TSize}\left(s_{2}\right)$.

In a similar way, we can show that permute $(O, I)$ is atom-terminating. ${ }^{3}$ However, permute $(I, O)$ is not atom-terminating.

The book on the Gödel language [14, page 81] shows a program that contains a clause, which in Prolog would be written as

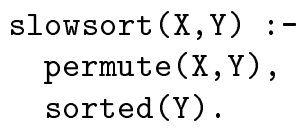

The meaning and the modes of the predicates should be obvious from their names, and there are delay declarations to ensure that derivations are inputconsuming. The predicate slowsort is not atom-terminating, but it could be made atom-terminating by replacing permute $(\mathrm{X}, \mathrm{Y})$ with permute $(\mathrm{Y}, \mathrm{X})$, so that permute is used in the mode in which it is atom-terminating.

Note that according to the Gödel specification, no guarantees are given about the selection rule that go beyond ensuring that derivations for the above program are input-consuming. Hence the program is not guaranteed to terminate even for a "well-behaved" query such as slowsort $([1,2], \mathrm{Y})$. Even though Hill and Lloyd do not claim that the program terminates, one would still expect it to do so. However, we can modify the program as stated, and guarantee that the modified program terminates using the method of this paper.

\footnotetext{
${ }^{3}$ Here we assume that the program is made well and nicely moded by interchanging the body atoms of the second clause.
} 
practice. Therefore level mappings such as the one used in the example will rarely be needed.

Consider again Def. 5.3. Given a clause $h \leftarrow a_{1}, \ldots, a_{n}$ and an atom $a_{i} \approx h$, we require $|h \theta|>\left|a_{i} \theta\right|$ for all grounding substitutions $\theta$, rather than only for $\theta$ such that $\left(a_{1}, \ldots, a_{i-1}\right) \theta$ is in a certain model of the program. This is of course a serious restriction. In Ex. 1.2, assuming mode permute $(I, O)$, there cannot exist a moded level mapping such that $\mid$ permute $(\mathrm{Y},[\mathrm{U} \mid \mathrm{X}]) \theta \mid>$ $\mid$ permute $(\mathrm{Z}, \mathrm{X}) \theta \mid$ for all $\theta$. That however is not surprising since permute $(I, O)$ is not atom-terminating.

Similarly, there cannot be a moded level mapping such that the usual recursive clause for quicksort, in the usual mode, is ICD-acceptable, although we conjecture that quicksort is atom-terminating. This shows a limitation of our method. The author is currently working on ways of overcoming this limitation, but the fact remains that many predicates are not atom-terminating.

\section{Applying the Method}

The requirement of input-consuming derivations merely reflects the very meaning of input: an atom must only consume its own input, not produce it. Thus if one accepts that (appropriately chosen) modes are useful for verification and reflect the programmer's intentions, then one should also accept this requirement and regard any violation of it as pathological. This does not exclude multiple modes, that is, the same program being used in a different mode at each run.

The requirement of input-consuming derivations is trivially met for LDderivations of a well moded query and program, ${ }^{4}$ since the leftmost atom in a well moded query is ground in its input positions. It can also be ensured by using delay declarations as in Gödel [14] that require the input arguments of an atom to be ground before this atom can be selected. Moreover, it might be ensured using guards as in GHC [25]. Finally, it can be ensured using delay declarations that check for partial instantiation of the input arguments, such as the block declarations of SICStus. Note that under certain conditions, delay declarations can ensure input-consuming derivations with respect to several, alternative modes [20, Chapter 7] [22].

Consequently, this paper is mainly aimed at logic programs with delay declarations, but unlike previous work $[2,16,17,18,19,22,23]$, abstracts from the details of particular delay constructs. We only assume what we see as the basic purpose of delay declarations: ensuring that derivations are input-consuming.

As we have said in the introduction, the class of predicates for which all input-consuming derivations terminate is quite limited. In an average program, some predicates are atom-terminating but some are not. In general,

\footnotetext{
${ }^{4}$ In particular, this means that it is met in Mercury [24].
} 
one has to make stronger assumptions about the selection rule. We sketch three ways in which the method presented here might be incorporated into a more comprehensive method for proving termination. This boils down to the question: how do we deal with predicates that are not atom-terminating?

The first way has actually been developed already [22]. We have previously considered atom-terminating predicates in a more concrete setting than here and called them robust predicates. The default left-to-right selection rule of most Prolog implementations is assumed. It is exploited that the textual position of atoms using robust predicates in clause bodies is irrelevant for termination. The other atoms must be placed such that the atoms producing their input occur earlier.

Secondly, we could build on a technique by Martin and King [18]. They consider coroutining derivations, but impose a bound on the depth of each sub-derivation by introducing auxiliary predicates with an additional argument that serves as depth counter. Applying the results of this paper, we only have to impose this depth bound for the predicates that are not atomterminating. For the atom-terminating predicates, we can save the overheads involved in this technique.

Thirdly, we could use delay declarations as they are provided for example in Gödel [14]. For the atom-terminating predicates, it is sufficient to check for partial instantiation of the input positions using a DELAY ... UNTIL NONVAR ... declaration. For the other predicates, it must be ensured that the input positions are ground using a DELAY ... UNTIL GROUND ... declaration. Note that according to its specification, Gödel does not guarantee a (default) left-toright selection rule, and therefore delay declarations are crucial for termination. Note also that a groundness test is usually more expensive than a test for partial instantiation. To the best of our knowledge, there has never been a systematic treatment of the question when GROUND declarations are needed, and when NONVAR declarations are sufficient.

\section{Discussion}

We have identified the class of predicates for which all input-consuming derivations are finite. An input-consuming derivation is a derivation where in each step, the input arguments of the selected atom are not instantiated. Predicates can be shown to be in that class using the notions of level mapping and acceptable clause [7, 10, 12].

Most previous approaches, including approaches for programs with delay declarations, can only show termination making stronger assumptions about the selection rule $[16,17,18]$. We have argued in the previous section that knowing the predicates that terminate under our weaker assumptions is useful even for programs where not all predicates have this property.

This paper builds on our own previous work [22], but attempts to formulate the results more abstractly, without getting involved in the details of particular delay constructs. For example, we previously imposed a restriction 
that all clause heads in a program must be input-linear, which is necessary so that block declarations can ensure input-consuming derivations. In this paper, we do not impose this restriction. Hence if input-consuming derivations can be ensured without imposing this restriction, say by using guards as in GHC [25], then the results of this paper could be applied to show termination.

We have claimed that most other approaches to termination rely on the idea that the size of an atom can be pinned down when the atom is selected. Technically, this usually means that the atom is bounded with respect to some level mapping $[4,6,12,18]$. There are exceptions though $[8,10]$, where termination can be shown for the query, say, append $([\mathrm{X}],[], \mathrm{Zs})$ using as level mapping the term size of the first argument, even though the term size of $[\mathrm{X}]$ is not bounded. However, the method only works for LD-derivations and relies on the fact that any future instantiation of $\mathrm{X}$ cannot affect the derivation for append $([\mathrm{X}],[], \mathrm{Zs})$. Therefore it is effectively possible to pin down the size of append $([\mathrm{X}],[], \mathrm{Zs})$.

In contrast, we show that under certain conditions, it is enough to rely on a relative decrease in the size of the selected atom, even though this size cannot be pinned down. This is crucial to show termination of derivations with coroutining. More precisely, we exploit that an atom in a query cannot proceed indefinitely unless it is repeatedly fed by some other atom occurring earlier in the query. This implies that every derivation for the query is finite.

Bezem [6] has identified the class of strongly terminating programs, which are programs that terminate under any selection rule. While it is shown that every total recursive function can be computed by a strongly terminating program, this does not change the fact that few existing programs are strongly terminating. Transformations are proposed for three example programs to make them strongly terminating, but the transformations are complicated and ad-hoc.

On the whole, there seems to be a strong reluctance to give up the idea that the size of an atom must be pinned down when the atom is selected. This is true even for Bezem [6]. It is also true for Marchiori and Teusink [17], who assume a local selection rule, that is a rule under which only most recently introduced atoms can be resolved in each step. Martin and King [18] achieve a similar effect by bounding the depth of the computation introducing auxiliary predicates. It is more difficult to assess Lüttringhaus-Kappel [16] since his contribution is mainly to generate delay declarations automatically rather than prove termination. ${ }^{5}$ However in some cases, the delay declarations that are generated require an argument of an atom to be a rigid list before that atom can be selected, which is similar to $[17,18]$. Such uses of delay declarations go well beyond ensuring that derivations are input-consuming.

None of the above approaches $[6,16,17,18]$ can formally show termination under the weak assumptions we make here, even for derivations as trivial as the one in Ex. 1.1. Apt and Luitjes [2] give conditions for the termination

\footnotetext{
${ }^{5}$ For the reader familiar with that work, it is not said how programs are shown to be safe.
} 
of append, but those are ad-hoc and do not address the general problem. Naish [19] gives heuristics to ensure termination, but no formal results.

We have assumed that queries are well and nicely moded, which means that the atoms in the query are ordered ${ }^{6}$ so that there is a left-to-right dataflow. As a topic for future work, we envisage to prove termination of programs where these conditions are relaxed, such as programs using layered modes [13]. We believe that the crucial idea will be the same as in this paper, namely that one must rely on a relative decrease in size of the selected atom in each derivation step, rather than an absolute one. Therefore this paper should provide a good basis for this extension.

\section{Acknowledgements}

The author would like to thank Florence Benoy for proofreading this paper, and Sandro Etalle and Pat Hill for some helpful comments. This work was funded by EPSRC Grant No. GR/K79635.

\section{References}

[1] K. R. Apt. From Logic Programming to Prolog. Prentice Hall, 1997.

[2] K. R. Apt and I. Luitjes. Verification of logic programs with delay declarations. In V. S. Alagar and M. Nivat, editors, Proceedings of AMAST'95, LNCS, Berlin, 1995. Springer-Verlag. Invited Lecture.

[3] K. R. Apt and D. Pedreschi. Studies in pure Prolog: Termination. In J. W. Lloyd, editor, Proceedings of the Symposium in Computational Logic, LNCS, pages 150-176. Springer-Verlag, 1990.

[4] K. R. Apt and D. Pedreschi. Modular termination proofs for logic and pure Prolog programs. In G. Levi, editor, Advances in Logic Programming Theory, pages 183-229. Oxford University Press, 1994.

[5] K. R. Apt and A. Pellegrini. On the occur-check free Prolog programs. ACM Transactions on Programming Languages and Systems, 16(3):687-726, 1994.

[6] M. Bezem. Strong termination of logic programs. Journal of Logic Programming, 15(1 \& 2):79-97, 1993.

[7] D. De Schreye and S. Decorte. Termination of logic programs: The never-ending story. Journal of Logic Programming, 19/20:199-260, 1994.

[8] D. De Schreye, K. Verschaetse, and M. Bruynooghe. A framework for analysing the termination of definite logic programs with respect to call patterns. In Proceedings of FGCS, pages 481-488. ICOT Tokyo, 1992.

[9] S. Decorte and D. De Schreye. Automatic inference of norms: A missing link in automatic termination analysis. In Proceedings of the 10th International Logic Programming Symposium, pages 420-436. MIT Press, 1993.

\footnotetext{
${ }^{6}$ Or more generally: can be ordered (see [20, Subsect. 5.3] or the discussion after Example 3.1).
} 
[10] S. Decorte and D. De Schreye. Termination analysis: Some practical properties of the norm and level mapping space. In J. Jaffar, editor, Proceedings of the 15th JICSLP, pages 235-249. MIT Press, 1998.

[11] P. Dembinski and J. Małuszyński. AND-parallelism with intelligent backtracking for annotated logic programs. In Proceedings of the 2nd International Logic Programming Symposium, pages 29-38. MIT Press, 1985.

[12] S. Etalle, A. Bossi, and N. Cocco. Termination of well-moded programs. Journal of Logic Programming, 38(2):243-257, 1999.

[13] S. Etalle and M. Gabbrielli. Layered modes. Journal of Logic Programming, 39:225-244, 1999.

[14] P. M. Hill and J. W. Lloyd. The Gödel Programming Language. MIT Press, 1994.

[15] J. W. Lloyd. Foundations of Logic Programming. Springer-Verlag, 1987.

[16] S. Lüttringhaus-Kappel. Control generation for logic programs. In D. S. Warren, editor, Proceedings of the 10th International Conference on Logic Programming, pages 478-495. MIT Press, 1993.

[17] E. Marchiori and F. Teusink. Proving termination of logic programs with delay declarations. In J. W. Lloyd, editor, Proceedings of the 12th International Logic Programming Symposium, pages 447-461. MIT Press, 1995.

[18] J. C. Martin and A. M. King. Generating efficient, terminating logic programs. In M. Bidoit and M. Dauchet, editors, Proceedings of TAPSOFT'97, LNCS, pages 273-284. Springer-Verlag, 1997.

[19] L. Naish. Coroutining and the construction of terminating logic programs. Technical Report 92/5, University of Melbourne, 1992.

[20] J.-G. Smaus. Modes and Types in Logic Programming. PhD thesis, University of Kent at Canterbury, September 1999. Draft available from www.cs.ukc.ac.uk/people/staff/jgs5/thesis.ps.

[21] J.-G. Smaus. Proving termination of input-consuming logic programs. Technical Report 10-99, Computing Laboratory, University of Kent at Canterbury, United Kingdom, 1999.

[22] J.-G. Smaus, P. M. Hill, and A. M. King. Termination of logic programs with block declarations running in several modes. In C. Palamidessi, editor, Proceedings of PLILP/ALP, LNCS. Springer-Verlag, 1998.

[23] J.-G. Smaus, P. M. Hill, and A. M. King. Preventing instantiation errors and loops for logic programs with multiple modes using block declarations. In P. Flener, editor, Proceedings of LOPSTR'98, LNCS. Springer-Verlag, 1999.

[24] Z. Somogyi, F. Henderson, and T. Conway. The execution algorithm of Mercury, an efficient purely declarative logic programming language. Journal of Logic Programming, 29(1-3), 1996.

[25] K. Ueda. Guarded Horn clauses. In E. Wada, editor, Proceedings of the 4th Japanese Conference on Logic Programming, LNCS, pages 168-179. SpringerVerlag, 1986. 